\title{
Clinicopathological significance of CD133 in lung cancer: A meta-analysis
}

\author{
YAOXI TAN, BO CHEN, WEI XU, WEIHONG ZHAO and JIANQING WU \\ Department of Geriatrics, The First Affiliated Hospital of Nanjing Medical University, Nanjing, \\ Jiangsu 210029, P.R. China
}

Received January 8, 2013; Accepted July 9, 2013

DOI: $10.3892 / \mathrm{mco} .2013 .195$

\begin{abstract}
CD133 is one of the most commonly used markers of lung cancer stem cells (CSCs), which are characterized by their ability for self-renewal and tumorigenicity. However, the clinical value and significance of CD133 in lung cancer remains controversial. Due to the limited size of the individual studies, the association between CD133 and the clinicopathological characteristics of lung cancer had not been fully elucidated. A meta-analysis based on published studies was performed with the aim of evaluating the effect of CD133 on the clinicopathological characteristics of lung cancer and to investigate the role of CSCs in the prognosis of lung cancer. A total of 15 eligible studies were included in this meta-analysis and our results indicated that a positive CD133 expression was significantly associated with poor differentiation and lymph node metastasis, although it was not associated with tumor stage or histological type. Therefore, CD133 may be considered as a prognostic maker of lung cancer. Further clinical studies, with larger patient samples, unified methods and cut-off levels to detect CD133 expression, classified by tumor stage, therapeutic schedule, follow-up time and survival events, are required to determine the role of CD133 in clinical application and the association between CD133 and the prognosis of lung cancer.
\end{abstract}

\section{Introduction}

Lung cancer remains the most common cause of cancer-related mortality worldwide (1). Patients affected by lung cancer exhibit a poor prognosis. Despite the significant advances in surgical techniques, chemotherapy and radiotherapy, the relapse rate is high and only a few patients achieve long-term survival, with

Correspondence to: Professor Jianqing Wu, Department of Geriatrics, The First Affiliated Hospital of Nanjing Medical University, 300 Guangzhou Road, Nanjing, Jiangsu 210029, P.R. China

E-mail: jwuny@njmu.edu.cn

Key words: lung cancer, cancer stem cells, CD133, clinicopathological characteristics, prognosis, meta-analysis an overall 5-year survival rate of $\sim 15 \%$ (2). Evidence supports the cancer stem cell (CSC) hypothesis, according to which CSCs may be responsible for tumor initiation, metastasis, recurrence and resistance to treatment $(3,4)$. Due to the characteristics of CSCs, the conventional therapies are unable to effectively eliminate these cells. The residual CSCs continue to proliferate, leading to the relapse of cancer. A variety of molecules have been investigated as putative markers of CSCs in malignancies, including lung cancer (5). Among the various markers, CD133 is one of the most commonly used. It is widely expressed in a number of malignancies, such as glioblastomas (6), hepatocellular (7), ovarian (8), colon (9) and lung (10) carcinomas.

A growing number of CD133-positive cancer cells have been identified in lung cancer $(11,12)$. Eramo et al (10) observed that a rare population of CD133-positive cancer stem-like cells were able to self-renew and generate an unlimited progeny of non-tumorigenic cells, whereas the CD133-negative cancer cells lacked this potential. However, the association between CD133 expression and the clinicopathological characteristics of lung cancer remains unknown. Attempts to elucidate the association between CD133-positive cancer cells and clinicopathological characteristics in previous studies yielded controversial results $(13,14)$. The limited sample availability resulted in discrepancies regarding the clinical significance determined by different CSCs studies. Therefore, we performed a systematic review of the literature with a meta-analysis to determine the association between CSCs marker CD133 and the clinicopathological characteristics of lung cancer and to investigate the role of CSCs in the prognosis of lung cancer.

\section{Materials and methods}

Literature search. Studies were identified via an electronic search through Medline, EMBASE and the China National Knowledge Infrastructure (CNKI) databases, using the key words 'lung cancer' and 'CD133', completed by the personal bibliography of two of the authors (Yaoxi Tan and Bo Chen). The bibliographies reported in all the identified studies were used to complete the study search. Review articles were scanned to identify additional eligible studies. The search was completed on November 15, 2012. To be eligible for inclusion in this systematic review, a study was required to meet the following criteria: i) it only included patients with primary lung cancer; 
Table I. Main characteristics of the 15 eligible studies.

\begin{tabular}{|c|c|c|c|c|c|c|c|}
\hline First author & Year & $\begin{array}{l}\text { Histological } \\
\text { type }\end{array}$ & Method & Cut-off level & $\begin{array}{l}\text { No. of } \\
\text { patients }\end{array}$ & $\begin{array}{c}\text { No. of } \\
\text { case groups }\end{array}$ & Refs. \\
\hline Shien & 2012 & NSCLC & IHC & $>1 \%$ staining & 30 & 9 & (23) \\
\hline Bertolini & 2009 & NSCLC & IHC & Strong staining & 58 & 32 & (13) \\
\hline Salnikov & 2010 & NSCLC & $\mathrm{IHC}$ & Any staining & 88 & 56 & $(28)$ \\
\hline Cortes-Dericks & 2012 & $\mathrm{AD}$ & RT-PCR & Median & 64 & 63 & (18) \\
\hline $\mathrm{Li}$ & 2011 & NSCLC & IHC & $>1 \%$ staining & 145 & 46 & $(22)$ \\
\hline Herpel & 2011 & NSCLC & $\begin{array}{c}\text { Tissue } \\
\text { microarray }\end{array}$ & $\begin{array}{l}\text { Diffuse expression } \\
\text { or distinct staining } \\
\text { in at least } 1 \text { out of } 4 \\
\text { tissue cores per sample }\end{array}$ & 86 & 13 & $(19)$ \\
\hline $\mathrm{Xu}$ & 2011 & NSCLC & IHC & Any staining & 103 & 51 & (14) \\
\hline Wei & 2008 & NSCLC & IHC & $>10 \%$ staining & 77 & 40 & $(26)$ \\
\hline $\mathrm{Li}$ & 2011 & N/E LC & IHC & $>10 \%$ staining & 90 & 44 & $(21)$ \\
\hline Yao & 2010 & $\mathrm{LC}$ & IHC & $>10 \%$ staining & 42 & 31 & (27) \\
\hline Lin & 2009 & SQ & IHC & Any staining & 54 & 27 & (29) \\
\hline $\mathrm{Gu}$ & 2010 & NSCLC & IHC & $>10 \%$ staining & 44 & 30 & (30) \\
\hline Cheng & 2010 & NSCLC & IHC & $>10 \%$ staining & 65 & 45 & (31) \\
\hline Sun & 2012 & NSCLC & IHC & $\begin{array}{c}\text { Method described } \\
\text { by Xu and Yang (33) }\end{array}$ & 67 & 42 & (32) \\
\hline Tirino & 2009 & NSCLC & $\begin{array}{c}\text { Flow } \\
\text { cytometry }\end{array}$ & NA & 89 & 64 & (20) \\
\hline
\end{tabular}

NSCLC, non-small-cell lung cancer; AD, adenocarcinoma; LC, lung cancer; SQ, squamous cell carcinoma; N/E LC, neuroendocrine lung cancer; IHC, immunohistochemistry; RT-PCR, reverse transcriptase-polymerase chain reaction; NA, not available.

ii) it investigated the association between CD133 and clinicopathological characteristics; iii) it was published as a full-text article in English or Chinese and; iv) it reported the number of CD133-positive and -negative patients. When duplicate studies were published, only the most recent or most informative was included in the analysis, to avoid overlap between cohorts.

Data extraction. The following information was extracted from each study: i) year of publication and first author's name; ii) sample size, test method and cut-off level; iii) tumor data including stage, grade, histological type and lymph node metastasis. Information was carefully extracted from all the eligible studies independently by two of the authors of the present study (Yaoxi Tan and Bo Chen). Differences in the extraction of data were assessed by a third investigator (Jianqing Wu).

Statistical analysis. To assess the association between CD133 and the clinicopathological characteristics of lung cancer including stage, grade, histological type and lymph node metastasis, odds ratios (ORs) with $95 \%$ confidence intervals (CIs) were calculated. The heterogeneity of combined ORs was initially evaluated by graphical examination of the forest plots. Statistical assessment was then performed using a $\chi^{2}$-based test of homogeneity and evaluation of the inconsistency index $\left(\mathrm{I}^{2}\right)$ statistic. The $\mathrm{I}^{2}$ statistic was defined as the percentage of variability due to heterogeneity rather than chance, with values $>50 \%$ representing the possibility of substantial heterogeneity (15). $\mathrm{P}<0.05$ was considered to indicate a statistically significant difference. If no obvious heterogeneity existed, the OR was calculated by the fixed-effects model (the Mantel-Haenszel method) and $\chi^{2}$ tests. Otherwise, the random-effects model (the DerSimonian-Laird method) was used. In addition, evidence of publication bias was determined using the Egger's (16) and Begg's methods (17). All the calculations were performed using the Stata statistical software version 12.0 (StataCorp, College Station, TX, USA).

\section{Results}

Study characteristics. In total, 15 studies published between 2008 and 2012 were selected for this systematic review. The study sample size ranged from 30 to 145 subjects. All studies investigated the association between CD133 and the clinicopathological characteristics of lung cancer. Cortes-Dericks et al (18) used reverse transcriptase-polymerase chain reaction (RT-PCR) to detect the expression of CD133, whereas Herpel et al (19) used tissue microarray, Tirino et al (20) used flow cytometry and the remaining studies used immunohistochemistry (IHC) with different cut-off levels. A total of 11 studies investigated non-small-cell lung cancer (NSCLC) alone, one study investigated adenocarcinoma and one squamous cell carcinoma. One study included adenocarcinoma, squamous cell carcinoma and small-cell lung cancer (SCLC). Li et al (21) conducted the study on patients with neuroendocrine lung cancer. The main characteristics of the 15 eligible publications are presented in Table I. 


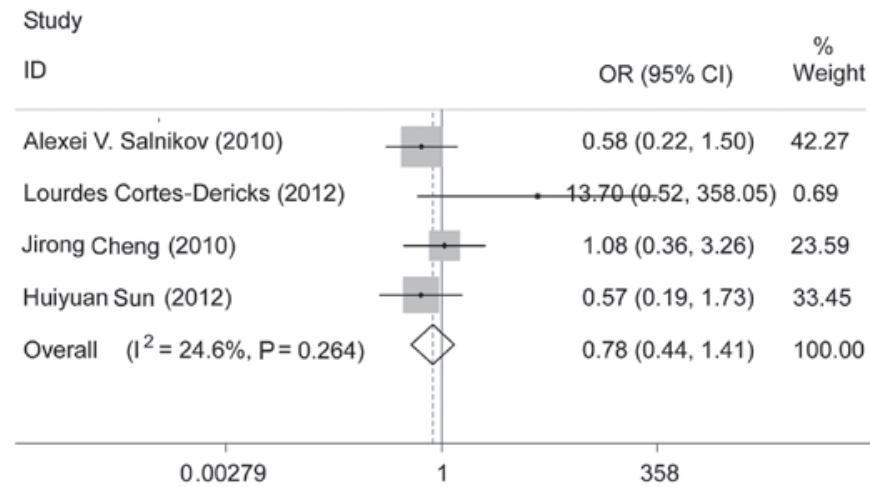

Figure 1. Correlation of CD133 with tumor stage.

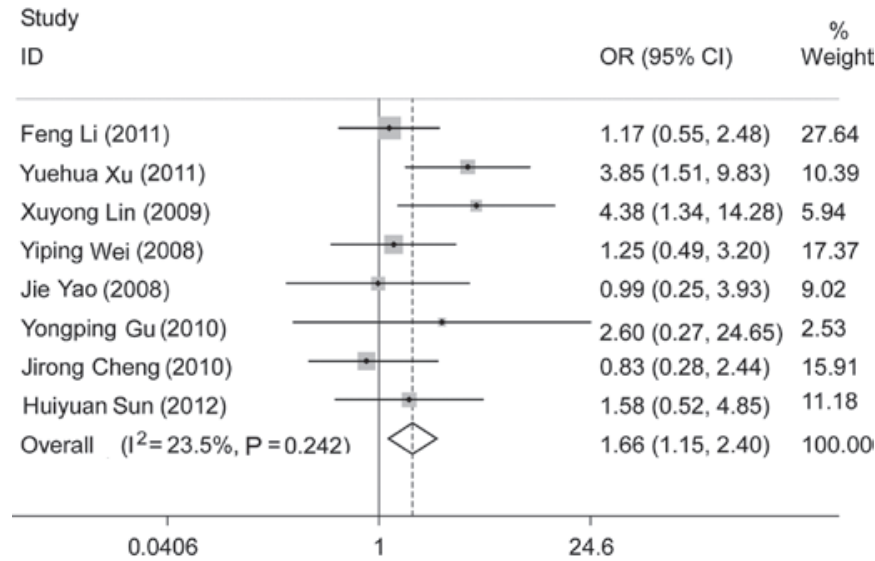

Figure 2. Correlation of CD133 with tumor differentiation.

\section{Main results of the meta-analysis}

Correlation of CD133 with tumor stage. Among the selected studies, 8 analyzed the association between CD133 and tumor stage. Cortes-Dericks et al (18) and Li et al (21) observed that positive CD133 expression exhibited a significant association with tumor stage. Of the 8 studies, one study (22) was limited to stage I and one study (19) assessed patients with stage I-II disease. Two studies $(13,21)$ were performed on stage I-IV lung cancer of different histological types. Excluding the four studies mentioned above, we performed a meta-analysis of the studies which investigated stage I-III cancer. A comparison of stage I-II to stage III revealed that there was no association between a positive CD133 expression and tumor stage (pooled $\mathrm{OR}=0.78$, 95\% CI: 0.44-1.41 and $\mathrm{P}=0.411$ ) (Fig. 1). There was no evident publication bias (Egger's test, $\mathrm{P}=0.089$ ), a finding also supported by the Begg's funnel plots (figure not shown).

Correlation of CD133 with tumor differentiation. Eleven studies investigated the association between CD133 and tumor differentiation. Three of those studies $(13,14,18)$ concluded that positive CD133 expression was associated with poorly differentiated tumors. Significant heterogeneity (pooled $\mathrm{OR}=1.17$, 95\% CI: 0.68-2.00 and $\mathrm{P}=0.567$; random effects, $\mathrm{I}^{2}=55.3 \%$ and $\mathrm{P}=0.013)$ existed when we analyzed all 11 studies. Excluding the studies using RT-PCR (18) and tissue microarray (19), we performed a subgroup analysis among the studies using IHC. However, significant heterogeneity (pooled $\mathrm{OR}=1.39$, 95\% CI: 0.81-2.37 and $\mathrm{P}=0.229$; random effects, $\mathrm{I}^{2}=52.2 \%$ and $\mathrm{P}=0.033$ ) was still evident. We excluded one study (13) which was conducted on European patients and analyzed the studies that investigated Asian patients using IHC. The comparison of poor to high tumor differentiation revealed that a positive CD133 expression was significantly correlated with poor differentiation (pooled OR=1.66, 95\% CI: 1.15-2.40 and $\mathrm{P}=0.006)$ without significant heterogeneity $\left(\mathrm{I}^{2}=23.5 \%\right.$ and $\mathrm{P}=0.242$ ) (Fig. 2). No evident publication bias existed (Egger's test, $\mathrm{P}=0.684$ ), a finding also supported by the Begg's funnel plots (figure not shown).

Correlation of CD133 with histological type. A total of 12 studies were found to be eligible for the analysis of the association between a positive CD133 expression and histological type. Of these, Bertolini et al (13) observed a correlation between a positive CD133 expression and adenocarcinoma. We excluded two more studies, one of which (18) investigated adenocarcinoma alone, while the other (21)

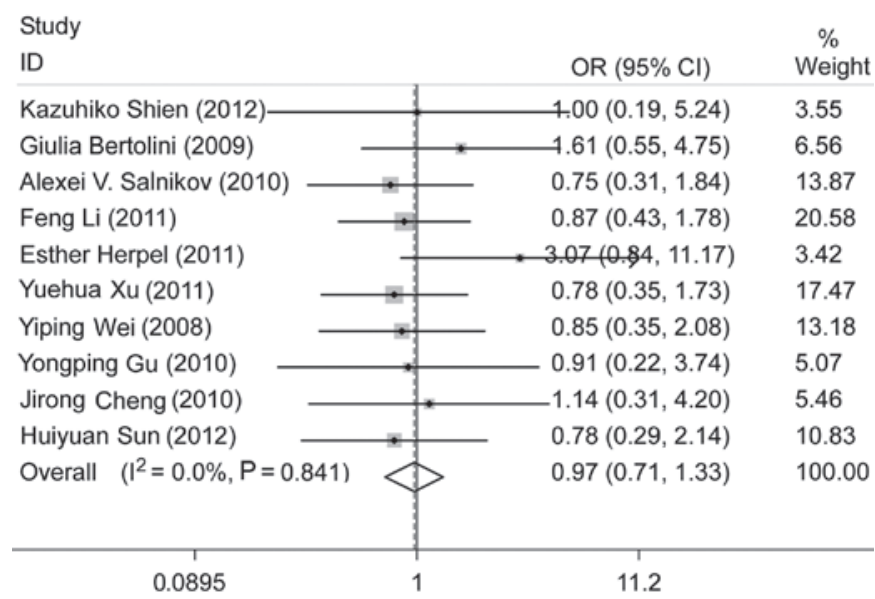

Figure 3. Correlation of CD133 with histological type.

investigated neuroendocrine lung cancer. We analyzed the remaining 9 studies which were conducted on patients with NSCLC. There was no significant association between positive CD133 expression and histological type (adenocarcinoma vs. non-adenocarcinoma), with pooled $\mathrm{OR}=0.97$, 95\% CI: 0.71-1.33 and $\mathrm{P}=0.86$ (Fig. 3). No evident publication bias existed (Egger's test, $\mathrm{P}=0.143$ ), a finding also supported by the Begg's funnel plots (figure not shown).

Correlation of CD133 with lymph node metastasis. Eleven publications investigated the association between CD133 expression and lymph node metastasis. One study (23), which was limited to patients with N2 or N3 NSCLC undergoing induction chemoradiotherapy (CRT), was excluded. We observed that CD133 was associated with nodal status (pooled $\mathrm{OR}=2.30,95 \%$ CI: $1.36-3.89$ and $\mathrm{P}=0.002$ ), with a positive CD133 expression in tumors with lymph node metastasis (N+ compared to N0) (Fig. 4). No evident publication bias existed (Egger's test, $\mathrm{P}=0.639$ ), a finding also supported by the Begg's funnel plots (figure not shown). However, there was significant heterogeneity. We performed a subgroup analysis among the studies that used IHC and observed that positive CD133 expression was associated with nodal status (pooled $\mathrm{OR}=2.98$, 95\% CI, 2.04-4.35 and $\mathrm{P}<0.001$ ), without significant heterogeneity (Fig. 5). 


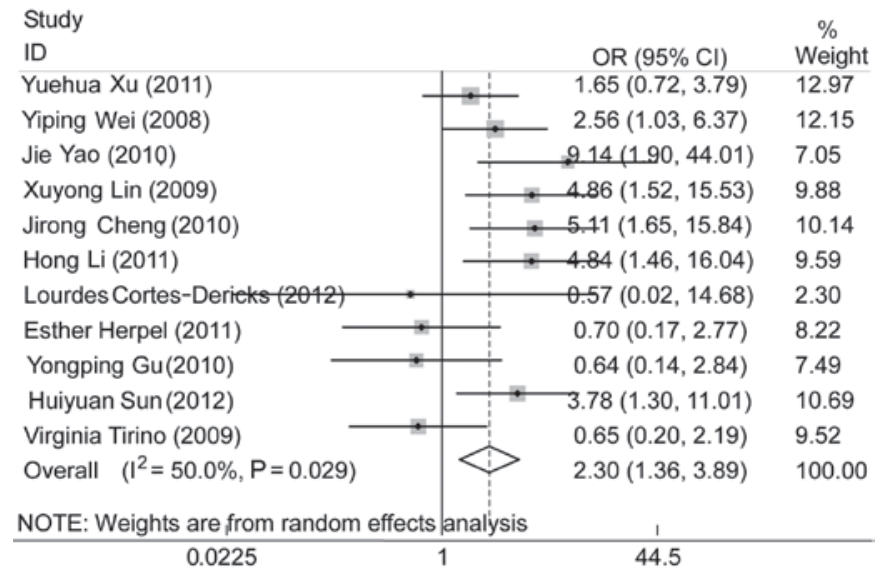

Figure 4. Correlation of CD133 with lymph node metastasis.

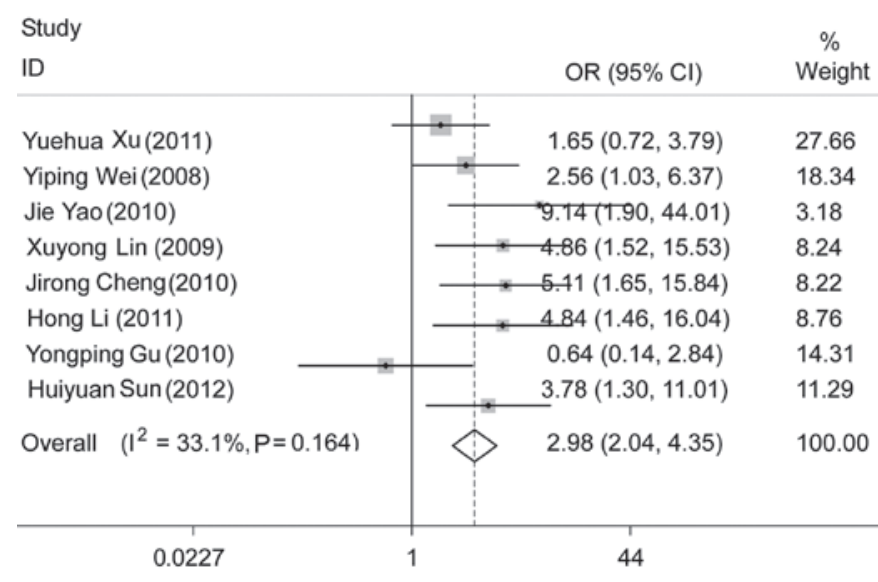

Figure 5. Subgroup analysis of the correlation of CD133 with lymph node metastasis.

\section{Discussion}

Accumulating evidence indicates that specific subpopulations of cancer cells with stem cell characteristics within the majority of tumors may be crucial in the pathogenesis of malignant tumors, including lung cancer. Several methods were used to identify and enrich CSCs from lung cancer, e.g., side populations and stem cell markers of normal tissue (24). CD133 is one of the most extensively used markers in lung cancer, although its function in lung cancer has not been fully elucidated. It remains controversial whether CD133 is associated with clinicopathological characteristics and prognosis of lung cancer. The aim of this current meta-analysis was to investigate the correlation between CD133 expression and the clinicopathological characteristics in patients with lung cancer, since therapeutic decisions are directly associated with clinicopathological characteristics, such as tumor type and differentiation. Our results indicated that a positive CD133 expression was significantly associated with differentiation and lymph node metastasis, although it was not associated with tumor stage or histological type.

Several studies $(14,18,21,23,25-27)$ reported that CD133 expression was correlated with poor prognosis in lung cancer, whereas other authors $(13,19,22,28)$ observed no such association. The patient populations varied among these studies. For example, Herpel et al (19) conducted the study on previously untreated stage I-II NSCLC patients, while Shien et al (23) investigated patients with locally advanced N2 or N3 NSCLC, who underwent induction CRT followed by surgery. The methods used to detect CD133 expression were also different, including IHC, RT-PCR and tissue microarray. Even among the studies using IHC, the cut-off level and the antibodies used varied widely. In addition, certain studies reported disease-free survival, whereas others reported overall survival with different follow-up times. Therefore, due to these considerable differences, we were not able to directly perform a systematic review to assess the correlation between CD133 expression and the prognosis of lung cancer.

The studies mentioned previously provided important information regarding the indirect prognostic value of CD133 and the therapy targeting CSCs in lung cancer. Eramo et al (10) observed that CD133-positive stem-like cancer cells were capable of self-renewal. The injection of immunocompromised mice with CD133-positive lung cancer cells readily generated tumor xenografts phenotypically identical to the original tumor, leading to the conclusion that CD133 was a reliable marker of lung cancer. We inferred that there was a larger percentage of CSCs in the majority of lung cancer tissue, with a higher number of CD133-positive lung cancer cells. One of the properties of CSCs was the ability to undergo asymmetrical division, leading to pluripotential differentiation and metastasis (28). This finding was consistent with the results of our meta-analysis, suggesting that a positive CD133 expression was clearly associated with poor tumor differentiation and lymph node metastasis. Poor differentiation and metastasis were significantly associated with poor survival of cancer. In brief, positive CD133 expression was most likely correlated with poor prognosis of lung cancer. It should also be noted that, since CD133 positivity was correlated with the expression of resistance-related proteins (28), the proportion of CSCs in patients who had received radiotherapy and/or chemotherapy was higher compared to that in patients who received surgery alone. As a result, it was not clear whether CD133 expression was of higher prognostic value in patients who received radiotherapy and/or chemotherapy. Further studies are required to elucidate the direct association between CD133 expression and the prognosis of lung cancer.

A meta-analysis is a quantitative approach in which individual study findings on the same topic are statistically integrated and analyzed. With more samples, the results of a meta-analysis are more reliable compared to those of a single study. However, the present meta-analysis had certain limitations. When analyzing whether CD133 expression was associated with tumor differentiation or lymph node metastasis, there was significant heterogeneity. The methods used to detect CD133 expression varied widely among the studies and heterogeneity was eliminated following exclusion of the studies that did not use IHC. Although IHC was the most commonly applied method, the cut-off level was defined differently among the studies. In addition, the IHC results were based on the primary antibody used and different antibodies were used by the eligible studies. The dilution of the antibody also varied, leading to differences in the sensitivity of the method, depending on the antibody concentration. Another factor was 
the effect of ethnicity. The majority of the studies included were conducted on Asian patients. There is the possibility that different ethnic groups exhibit differences in CD133 expression, leading to heterogeneity and bias.

Although there was no evident publication bias in this meta-analysis, we were not able to completely exclude biases. For example, the study was restricted to studies published in English and Chinese, which may lead to bias.

In conclusion, our meta-analysis suggests that CD133 expression is significantly associated with poor differentiation and lymph node metastasis in lung cancer. CD133 expression is most likely correlated with poor prognosis of lung cancer. However, further studies are required, with larger patient samples, unified methods and cut-off levels to detect CD133 expression, classified by tumor stage, therapeutic schedule, follow-up time and survival events, to confirm the findings of the present meta-analysis.

\section{Acknowledgements}

This study was a project funded by the Priority Academic Program Development of Jiangsu Higher Education Institutions and also supported by the National Natural Science Foundation of China (no. 81272602).

\section{References}

1. Jemal A, Siegel R, Xu J and Ward E: Cancer statistics. CA Cancer J Clin 60: 277-300, 2010.

2. Alberg AJ, Ford JG and Samet JM; American College of Chest Physicians: Epidemiology of lung cancer: ACCP evidence-based clinical practice guidelines (2nd edition). Chest 132 (Suppl 3): S29-S55, 2007.

3. Dontu G, Liu S and Wicha MS: Stem cells in mammary development and carcinogenesis: implications for prevention and treatment. Stem Cell Rev 1: 207-213, 2005.

4. Donnenberg VS and Donnenberg AD: Multiple drug resistance in cancer revisited: the cancer stem cell hypothesis. J Clin Pharmacol 45: 872-877, 2005.

5. Toyooka S, Mitsudomi T, Soh J, et al: Molecular oncology of lung cancer. Gen Thorac Cardiovasc Surg 59: 527-537, 2011.

6. Singh SK, Clarke ID, Terasaki M, Bonn VE, Hawkins C, Squire J and Dirks PB: Identification of a cancer stem cell in human brain tumors. Cancer Res 63: 5821-5828, 2003.

7. Suetsugu A, Nagaki M, Aoki H, Motohashi T, Kunisada T and Moriwaki $\mathrm{H}$ : Characterization of $\mathrm{CD} 133^{+}$hepatocellular carcinoma cells as cancer stem/progenitor cells. Biochem Biophys Res Commun 351: 820-824, 2006.

8. Ferrandina G, Bonanno G, Pierelli L, et al: Expression of CD133-1 and CD133-2 in ovarian cancer. Int J Gynecol Cancer 18: 506-514, 2008

9. Kojima M, Ishii G, Atsumi N, Fujii S, Saito N and Ochiai A: Immunohistochemical detection of CD133 expression in colorectal cancer: a clinicopathological study. Cancer Sci 99: 1578-1583, 2008.

10. Eramo A, Lotti F, Sette G, et al: Identification and expansion of the tumorigenic lung cancer stem cell population. Cell Death Differ 15: 504-514, 2008.

11. Janikova M, Skarda J, Dziechciarkova M, et al: Identification

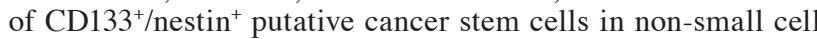
lung cancer. Biomed Pap Med Fac Univ Palacky Olomouc Czech Repub 154: 321-326, 2010.

12. Cui F, Wang J, Chen D and Chen YJ: CD133 is a temporary marker of cancer stem cells in small cell lung cancer, but not in non-small cell lung cancer. Oncol Rep 25: 701-708, 2011.
13. Bertolini G, Roz L, Perego P, et al: Highly tumorigenic lung cancer $\mathrm{CD}_{133^{+}}$cells display stem-like features and are spared by cisplatin treatment. Proc Natl Acad Sci USA 106: 16281-16286, 2009.

14. Xu YH, Wang JM, Zhang GB and Hu HC: Expression and clinical significance of CD133 and B7-H4 in non-small cell lung cancer. Jiangsu Med J 4: 412-415, 2011.

15. Higgins JP, Thompson SG, Deeks JJ and Altman DG: Measuring inconsistency in meta-analyses. BMJ 327: 557-560, 2003.

16. Egger M, Davey Smith G, Schneider M and Minder C: Bias in meta-analysis detected by a simple, graphical test. BMJ 315: 629-634, 1997.

17. Begg CB and Mazumdar M: Operating characteristics of a rank correlation test for publication bias. Biometrics 50: 1088-1101, 1994.

18. Cortes-Dericks L, Galetta D, Spaggiari L, Schmid RA and Karoubi G: High expression of octamer-binding transcription factor 4A, prominin-1 and aldehyde dehydrogenase strongly indicates involvement in the initiation of lung adenocarcinoma resulting in shorter disease-free intervals. Eur J Cardiothorac Surg 41: e173-e181, 2012.

19. Herpel E, Jensen K, Muley T, et al: The cancer stem cell antigens CD133, BCRP1/ABCG2 and CD117/c-KIT are not associated with prognosis in resected early-stage non-small cell lung cancer. Anticancer Res 31: 4491-4500, 2011.

20. Tirino V, Camerlingo R, Franco R, et al: The role of CD133 in the identification and characterisation of tumour-initiating cells in non-small-cell lung cancer. Eur J Cardiothorac Surg 36: 446-453, 2009.

21. Li H, Wang Y, Yu L and Zhao P: Clinicopathological significance of expression of CD133 protein in neuroendocrine lung carcinoma tissues. Chin J Cancer Prev Treat 18: 29-31, 2011.

22. Li F, Zeng $\mathrm{H}$ and Ying K: The combination of stem cell markers CD133 and ABCG2 predicts relapse in stage I non-small cell lung carcinomas. Med Oncol 28: 1458-1462, 2011.

23. Shien K, Toyooka S, Ichimura K, et al: Prognostic impact of cancer stem cell-related markers in non-small cell lung cancer patients treated with induction chemoradiotherapy. Lung Cancer 77: 162-167, 2012.

24. Rivera C, Rivera S, Loriot Y, Vozenin MC and Deutsch E: Lung cancer stem cell: new insights on experimental models and preclinical data. J Oncol 2011: 549181, 2011.

25. Woo T, Okudela K, Mitsui H, et al: Prognostic value of CD133 expression in stage I lung adenocarcinomas. Int J Clin Exp Pathol 4: 32-42, 2010.

26. Wei YP, Wang M, Hua P, et al: Expression of tumor stem cell marker CD133 in non-small cell lung carcinoma and its clinical significance. J Sun Yat-Sen Univ (Med Sci) 29: 312-316, 2008.

27. Yao J, Wang ZG, Tong XW, Li ZJ and Yu ZG: Expression of tumor stem cell marker CD133 and CD44 in original tumor tissues and metastatic lymph nodes of lung cancer. Med J Natl Defend Force Southwest China 20: 1300-1303, 2010

28. Salnikov AV, Gladkich J, Moldenhauer G, Volm M, Mattern J and Herr I: CD133 is indicative for a resistance phenotype but does not represent a prognostic marker for survival of non-small cell lung cancer patients. Int J Cancer 126: 950-958, 2010.

29. Lin X, Liu S, Liu N, Yang X, Xu H and Wang E: Expression and significance of stem cell markers CK19, Notch3, CD133, P75NTR, STRO-1 and ABCG2 in pulmonary squamous carcinomas. Zhongguo Fe 12: 316-321, 2009 (In Chinese).

30. Gu YP, Sun MM, Gu LQ, Zhang H and Xie F: Expression and significance of cancer stem cell marker CD133, ABCG2 and p75NTR in non-small cell lung carcinoma. Suzhou Univ J Med Sci 30: 513-516, 2010

31. Cheng JR, Wang SQ, Zu MRT and Zou J: Expressions of CD133 and CD105 in lung cancer tissue and their clinical significance. Tumor 30: 334-337, 2010.

32. Sun HY, Yang M, Zheng MJ, Ren ZJ and Liu H: Expression of CD133 and ALDH1 in non-small cell lung cancer and their clinical significance. J Clin Exp Pathol 28: 813-815, 2012.

33. Xu LZ and Yang WT: The criteria of immunohistochemical reaction results. China Oncol 6: 229-231, 1996. 